\title{
On the radiation driven alignment of dust grains: Detection of the polarization hole in a starless core ${ }^{\star}, \star \star$
}

\author{
F. O. Alves ${ }^{1, \star \star \star}$, P. Frau ${ }^{2,3}$, J. M. Girart ${ }^{4}$, G. A. P. Franco ${ }^{5}$, F. P. Santos ${ }^{6}$, and H. Wiesemeyer ${ }^{7}$ \\ 1 Argelander-Institut für Astronomie, Auf dem Hügel 71, 53121 Bonn, Germany \\ e-mail: falves@astro.uni-bonn.de \\ 2 Instituto de Ciencia de Materiales de Madrid (CSIC), Sor Juana Inés de la Cruz 3, 28049 Madrid, Spain \\ 3 Observatorio Astronómico Nacional, Alfonso XII 3, 28014 Madrid, Spain \\ ${ }^{4}$ Institut de Ciències de l'Espai (CSIC-IEEC), Campus UAB, Facultat de Ciències, C5 par 2a 08193 Bellaterra, Catalunya, Spain \\ 5 Departamento de Física - ICEx - UFMG, Caixa Postal 702, 30.123-970 Belo Horizonte, Brazil \\ 6 Department of Physics and Astronomy, Northwestern University, 2145 Sheridan Road, Evanston, IL 60208, USA \\ 7 Max-Planck-Institut für Radioastronomie, Auf dem Hügel 69, 53121 Bonn, Germany
}

Received 25 July 2014 / Accepted 21 August 2014

\begin{abstract}
Aims. We aim to investigate the polarization properties of a starless core in an early evolutionary stage. Linear polarization data reveal the properties of the dust grains in the distinct phases of the interstellar medium. Our goal is to investigate how the polarization degree and angle correlate with the cloud and core gas.

Methods. We use optical, near infrared, and submillimeter polarization observations on the starless object Pipe-109 in the Pipe nebula. Our data cover a physical scale range of 0.08 to $0.4 \mathrm{pc}$, comprising the dense gas, envelope, and the surrounding cloud.

Results. The cloud polarization is well traced by the optical data. The near infrared polarization is produced by a mixed population of grains from the core border and the cloud gas. The optical and near infrared polarization toward the cloud reaches the maximum possible value and saturates with respect to the visual extinction. The core polarization is predominantly traced by the submillimeter data and has a steep decrease with respect to the visual extinction. Modeling of the submillimeter polarization indicates a magnetic field main direction projected onto the plane-of-sky and loss of grain alignment for densities higher than $6 \times 10^{4} \mathrm{~cm}^{-3}$ (or $A_{V}>$ $30 \mathrm{mag})$.

Conclusions. The object is immersed in a magnetized medium with a very ordered magnetic field. The absence of internal source of radiation significantly affects the polarization efficiencies in the core, creating a polarization hole at the center of the starless core. This result supports the theory of dust grain alignment via radiative torques
\end{abstract}

Key words. stars: formation - ISM: magnetic fields - techniques: polarimetric - ISM: individual objects: Core 109

\section{Introduction}

Polarimetry is a unique technique to study the physical properties of dust grains. Magnetic fields and the interstellar radiation field are known to interact with dust grains, producing polarized light (Hall 1949; Hiltner 1949). The properties of the dust cloud, such as temperature and volume density, strongly affect the polarization power of grains (e.g., Serkowski et al. 1975; Goodman et al. 1995). In addition, polarimetry is a useful tool to characterize the magnetic field morphology. For example, linear polarization observations across a wide range of frequencies reveal the morphologic changes on the magnetic field topology from molecular clouds down to cloud cores (e.g., Alves et al. 2011, 2012).

\footnotetext{
* Based on data acquired with the Atacama Pathfinder Experiment (APEX) and the $1.6 \mathrm{~m}$ telescope at Observatorio do Pico dos Dias (LNA/MCTI).

$\star \star$ The data are only available at the CDS via anonymous ftp to cdsarc.u-strasbg.fr (130.79.128.5) or via

http://cdsarc.u-strasbg.fr/viz-bin/qcat?J/A+A/569/L1

$\star \star \star$ Present address: Max-Planck-Institut für extraterrestrische Physik, Giessenbachstrasse 1, 85748, Garching, Germany; e-mail: falves@mpe.mpg.de
}

This paper is focused on the very early stages of star formation in a highly magnetized environment. Our goal is to investigate the polarization properties of a textbook pre-stellar object, Pipe-109 ${ }^{1}$. This source is a starless core located in a pristine cloud, the Pipe nebula (Alves et al. 2007, 2008; Forbrich et al. 2009; Franco et al. 2010; Frau et al. 2010, 2012b). The object has a mass of $\sim 4 M_{\odot}$ and the chemistry of a typical evolved pre-stellar core (Frau et al. 2012a).

Polarization observations of starless cores are scarce. Only a handful of relevant dust polarization data of this class of objects have been published (Ward-Thompson et al. 2000, 2009; Crutcher et al. 2004; Nutter et al. 2004; Ward-Thompson et al. 2009). In this paper, we show combined optical, near infrared (near-IR) and submillimeter (submm) polarimetric data of a starless core for the first time. In all cases, we assume that the polarization is produced by aspherical dust grains aligned perpendicular to the ambient magnetic field (for a detailed review of the mechanisms of grain alignment, refer to Lazarian 2007). Optical and near-IR polarization are produced by differential absorption

\footnotetext{
Here, we follow the core numbering of Rathborne et al. (2008). It is also known as the dark globule FeSt 1-457 in the catalog of Feitzinger $\&$ Stuewe (1984) and is comprised by field 40 in the catalog of Franco et al. (2010).
} 
of the background radiation by the aligned grains. On the other hand, submm polarization is produced by the thermal continuum emission from the aligned dust grains. Therefore, the plane-ofsky component of the magnetic field is parallel to optical/nearIR polarization maps and perpendicular to submm polarization maps. Our submm data represent one of the few strong detections of dust continuum polarized flux toward starless cores.

\section{Observations}

\subsection{Optical/near infrared observations}

The optical data were obtained in $R$-band $(6474 \AA)$ and a detailed description of the observations and data reduction is found in Franco et al. (2010). The near-IR ( $H$-band) linear polarization observations were conducted at the Observatório do Pico dos Dias/Laboratório Nacional de Astrofísica (OPD/LNA, Brazil) using IAGPOL, the IAG imaging polarimeter mounted on the $1.6 \mathrm{~m}$ telescope. For a full description of the polarimeter, see Magalhães et al. (1996). The images were gathered using the CamIV infrared camera, which is based on a HAWAII detector of $1024 \times 1024$ pixels and $18.5 \mu \mathrm{m} /$ pixel that yields a plate scale of $0.25 /$ pixel. Sixty dithered images, following a five-dot pattern $(12 \times 5$ positions), were obtained for each of eight waveplate positions separated by 22.5 . The exposure time for each image was of $10 \mathrm{~s}$, totaling a combined exposure time of $600 \mathrm{~s}$ per waveplate position. The reference direction of the polarizer was determined by observing polarized standard stars. Polarization degree and position angle were obtained for 700 stars, almost $45 \%$ of which have $P / \sigma_{P} \geq 10$.

\subsection{Submm observations}

The submm polarization observations were performed with the PolKa continuum polarimeter at the APEX $12 \mathrm{~m}$ telescope. The polarimeter is operated with the LABOCA bolometer at $345 \mathrm{GHz}$ and uses a rotating half-wave plate as polarization modulator (for a detailed description of the instrument, see Siringo et al. 2004, 2012; Wiesemeyer et al. 2014). The main beam of the telescope at this wavelength is $\sim 20^{\prime \prime}$. There was a total of 600 scans of $2.5 \mathrm{~min}$ each, leading to a $25 \mathrm{~h}$ on-source time. Each scan consists of four subscans with a spiral stroke pattern with each centered on a different position. The mean zenith opacity was 0.2 (i.e., good weather conditions). The level of instrumental polarization of our data is about $0.10 \pm 0.04 \%$ toward the peak of emission, and it was determined by observing Uranus. The data discussed below is corrected by instrumental polarization. The final submm map has an rms noise of $\sim 5 \mathrm{mJy} /$ beam.

\section{Results}

Our results are exhibited in Fig. 1. We have used the visual extinction map of Román-Zúñiga et al. (2010), which accurately traces the gas column density of the core. It ranges from a few magnitudes toward the diffuse gas around the core up to $\sim 45 \mathrm{mag}$ at the core center. The upper panels of Fig. 1 show how the polarization degree and position angle of the magnetic field (PA) for the three bands vary with the visual extinction $\left(A_{V}\right)$. The subsequent discussion considers only polarization data whose signalto-noise ratio $P / \sigma_{P}$ is better than 10 (for optical and near-IR) and 3 (for the submm).

The submm emission has peak intensity of $224 \pm$ $5 \mathrm{mJy} /$ beam and traces only the region with $A_{V}$ higher
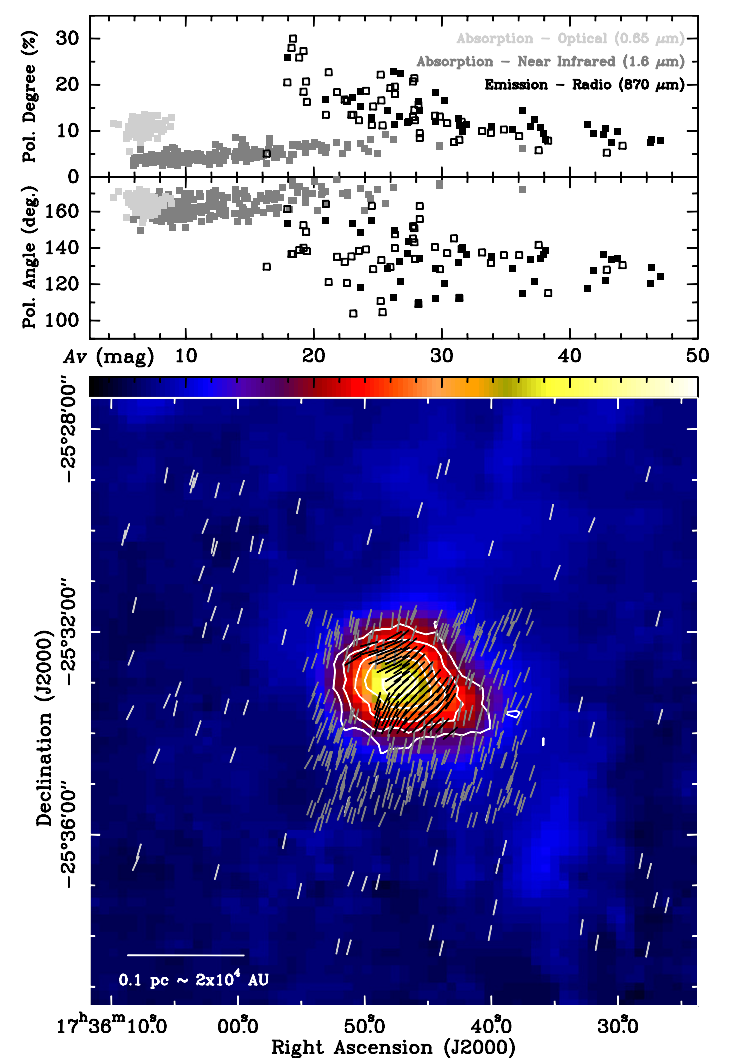

Fig. 1. Multi-wavelength polarimetry toward Pipe-109: in the lower panel, optical (light grey), near-IR (grey), and submm (black) polarization vectors are plotted over the visual extinction map of Román-Zúñiga et al. (2010). The vectors are scaled to the same size. The white contours are the $870 \mu \mathrm{m}$ dust continuum emission detected by PolKa. The contours are 10, 20, 30, 40, and 50 times the rms of the map $\left(\sim 5 \mathrm{mJy}\right.$ beam $\left.^{-1}\right)$. The submm vectors are rotated by $90^{\circ}$ to show the magnetic field direction. The dependence of the polarization degree and polarization angle of the three wavelengths with respect to the visual extinction is shown at the upper panels. Light grey and grey squares refer to the optical and near-IR data, respectively. Filled black squares and open squares refer to the submm data at $P / \sigma_{P}>3$ and 2, respectively.

than 10 mag (mag). We note that a combined Bonnor-Ebert fit to the visual extinction and $1.2 \mathrm{~mm}$ continuum maps shows that the Pipe-109 starts to contribute to the visual extinction at $\simeq 9.5 \mathrm{mag}$ (Frau et al., in prep.) with lower values being associated only to the cloud. Thus, the submm map is sensitive mainly to the starless core. The submm dust emission is optically thin: assuming a dust temperature of $T_{\text {dust }} \sim 9.5 \mathrm{~K}$ (Rathborne et al. 2008), we obtain a dust opacity of $\sim 10^{-3}$. The weighted mean polarization is $\sim 11 \%$ with maximum polarized flux of $\sim 13 \mathrm{mJy} / \mathrm{beam}$. Because of the higher sensitivity needed, the submm polarization is sensitive to $A_{V} \gtrsim 18 \mathrm{mag}$. The top panel of Fig. 1 shows that the polarization degree clearly decreases with the visual extinction: it goes from $20-30 \%$ at $A_{V} \sim 18 \mathrm{mag}$ down to $8 \%$ at the highest visual extinction, $45 \mathrm{mag}$. The magnetic field lines are relatively uniform with a weighted mean position angle of $130^{\circ}$ (counted from north to east direction) with a standard deviation of $\sim 12^{\circ}$. The magnetic field shows more structure at lower extinctions, as inferred by the increase on PA dispersion for $A_{V}<30$ mag ( $A_{V}-$ PA panel of Fig. 1$)$.

The near-IR data is sensitive to $A_{V}$ in the 6-36 mag, whereas the optical data traces the lowest visual extinction of the observed field, 4-9 mag. The weighted mean polarization degree for the optical and near-IR is $11 \%$ and $4 \%$, respectively. The 


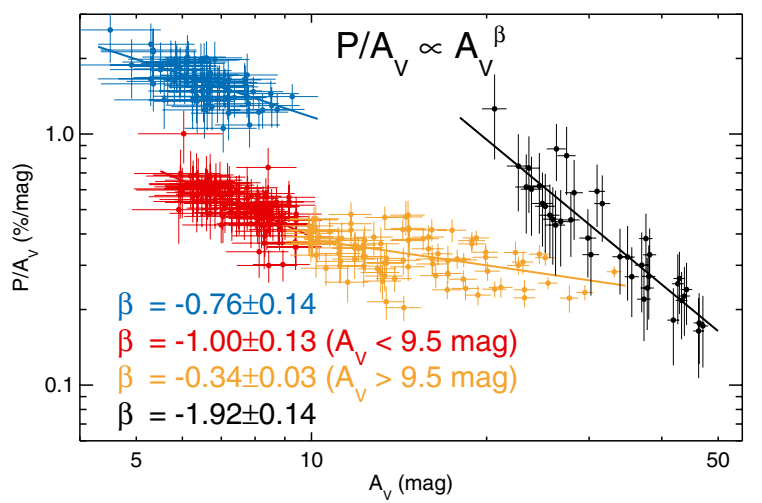

Fig. 2. Polarization efficiency $\left(P / A_{V}\right)$ as a function of the visual extinction $A_{V}$ for the three wavelengths. The blue dots are the optical data, red dots are near-IR for $A_{V}<9.5 \mathrm{mag}$, orange dots are near-IR data for $A_{V}>9.5 \mathrm{mag}$ and black dots are the submm data. The lines show the linear fit in the log-log basis for the aforementioned four different data.

near-IR polarization degree exhibits a smooth but clear increase at increasing visual extinction, especially at $A_{V} \gtrsim 10$ mag, reaching polarization degrees close to $10 \%$ at $A_{V} \simeq 25$ mag. Both data trace a very uniform magnetic field pattern, specially at low visual extinction (previously reported by Franco et al. 2010). Thus, the optical and near-IR data have a comparable weighted PA of $\sim 168^{\circ}$ and $163^{\circ}$, respectively. Their standard deviations are only $4^{\circ}$ and $5^{\circ}$. The remarkable uniformity of the optical and near-IR data can be also seen in the $A_{V}-\mathrm{PA}$ plot.

A noticeable result of Fig. 1 is the clear distinction between the two polarization regimes, absorption and emission. First, the mean magnetic field direction shown in the submm map, tracing the core's field, is clearly bended by $\simeq 35^{\circ}$ with respect to the optical and near-IR data, which mostly trace the cloud magnetic field. The near-IR and submm polarization data overlap in the region with a visual extinction in the 18-30 mag range. In this range, the observed discrepancy between the magnetic field directions of the near-IR and submm data is clearly seen in the northern and southwestern section of the core. However, they appear to be consistent in the southeastern and eastern parts of the core. Second, the polarization degree dependence with respect to the visual extinction shows an opposite behavior for the polarization data seen in absorption and emission. While the polarization degree is almost constant with respect to the visual extinction for the optical and near-IR data for $A_{V} \lesssim 10$ mag and then smoothly increases gradually up to $A_{V} \sim 20 \mathrm{mag}$, the submm polarization degree decreases dramatically, especially in the near-IR and submm overlapping range.

\section{The cloud and core polarization regimes}

To better understand the observed polarization properties, we have computed the polarization efficiency for the three bands with respect to the interstellar extinction map. The polarization efficiency, defined as the ratio between polarization degree and the visual extinction $\left(P / A_{V}\right)$, determines the polarizing power of the dust grains. This is shown in Fig. 2. We adopted a conservative uncertainty of 1 mag for the extinction map, which is higher than the value reported by Román-Zúñiga et al. (2010). Figure 2 shows that the polarization efficiency decreases in all three wavelengths but with different slopes. Thus, we performed a linear fit on a log-log basis for the different wavelengths. The optical polarization, produced uniquely by the cloud, shows a polarization efficiency slope of $-0.76 \pm 0.14$ with respect to the extinction. This is consistent with a regime where the polarization is almost saturated (e.g., Arce et al. 1998). The near-IR data have a clear breakpoint at $\simeq 10 \mathrm{mag}$, which is noticeably the point where the core starts to contribute. Below this value, where the near-IR arises only from the cloud, the slope is closer to full saturation than the optical data and is consistent with previous observations in other dark clouds (Goodman et al. 1995). However, above $\sim 10 \mathrm{mag}$, the slope is shallower, $-0.34 \pm 0.03$. This implies that $P \propto A_{V}^{0.66}$. This power law is similar to the one found in the dense regions of Perseus and Taurus (Whittet et al. 2008; Chapman et al. 2011) and is the predicted for the case of grain alignment mechanism via radiative torques (Dolginov \& Mytrophanov 1976; Draine \& Weingartner 1996, 1997; Lazarian $\&$ Hoang 2007). The change of the slope suggests that the nearIR polarization is produced by a mixed population of grains from both the cloud and the starless core for $A_{V}>10 \mathrm{mag}$ and that the core grains are more efficiently aligned than the cloud grains. Indeed, Goodman et al. (1995) proposed that the dust population in cores and their envelopes have a distinct size distribution, asymmetry level, and chemical composition.

The submm polarization efficiency, which arises uniquely from the core, falls with $\sim-1.9$ with respect to $A_{V}$, which suggests strong depolarization. Since the submm polarization shows an ordered field, the decrease in polarization efficiency cannot be fully ascribed to turbulence in the cloud. Molecular emission from dense gas tracers at the center of the core shows linewidths of $0.2-0.4 \mathrm{~km} \mathrm{~s}^{-1}$, implying subsonic non-thermal gas motions (Frau et al. 2010, 2012b). Since the radiative torque mechanism requires a radiation field, the submm depolarization is thus likely produced by a decrease on the grain alignment efficiency due to the lack of internal (no protostar) and external (absorbed UV photons) radiation. This is tested in the following section.

A puzzling issue is the discrepancy on the polarization efficiency regimes in the overlap region between the near-IR and submm polarization. There is also a discrepancy on the derived magnetic field direction (toward the north) between the two bands. The distinct slopes for the overlapping extinction range strongly suggests that there are two populations of dust grains within the core: one population is more sensitive to the near-IR background radiation than the other. They possibly respond to the external radiation in a different way, leading to distinct grain alignment regimes (as we see in Fig. 2). In addition, the discontinuity in magnetic field direction may be due to physical asymmetries of the core, where the two populations are unevenly distributed over the outer layers of the core and are therefore exposed differently to external ultraviolet (UV) radiation. Preferential grain alignment due to inhomogeneities in the cloud was previously reported by other groups (e.g., Whittet et al. 2008; Andersson et al. 2011) and could be the case here.

\section{The core's depolarization critical density}

We made use of a theoretical model to understand the origin of the submm dust polarization and its relationship to the volume density of Pipe-109. The source is likely to be on the verge of collapse, and hence, a good approximation is the initial pivotal stage used in core collapse simulations. The works by Li \& Shu (1996) and Allen et al. (2003a,b) match the observational properties of Pipe-109. They describe the initial stage of the magnetic field for an isolated, marginally supercritical, slightly oblate spheroid under ideal-MHD conditions.

To generate synthetic maps comparable to the data, we used the DustPol module (Padovani et al. 2012) to compute the polarization emission expected for the core under the previous 


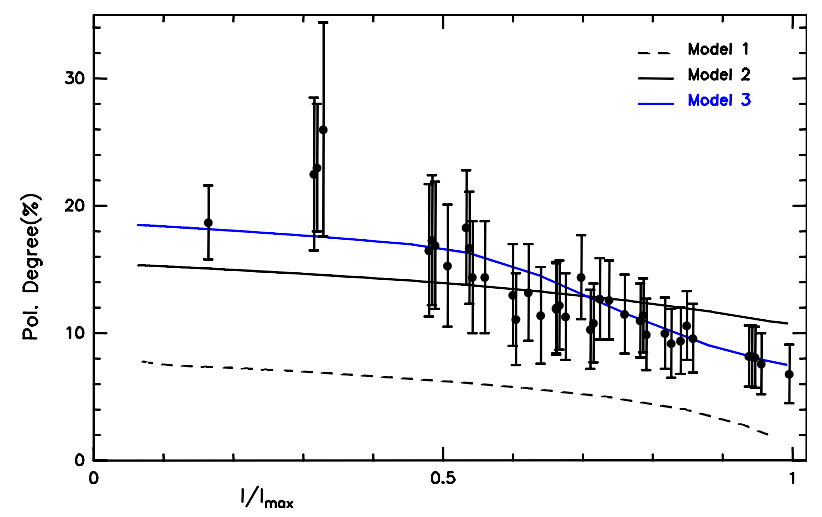

Fig. 3. Submillimeter polarization degree as a function of the submillimeter intensity normalized to its peak. The dots represent the PolKa data. Models 1 and 2: magnetized core with constant polarization efficiency $(\alpha=0.15)$ and with a magnetic field direction with respect to the line of sight of $45^{\circ}$ and $90^{\circ}$ (edge-on), respectively. Model 1 clearly underestimates the polarization degree. Model 3: edge-on magnetic field with $\alpha=0.18$ for densities $n_{\left(\mathrm{H}_{2}\right)}<6 \times 10^{4} \mathrm{~cm}^{-3}$.

assumptions. Initially, we tested the case of a constant polarizing efficiency parameter $\alpha$, which includes the absorption cross section and the alignment efficiency (Fiege \& Pudritz 2000). By adopting the commonly used value of $\alpha=0.15$ (e.g., Frau et al. 2011; Padovani et al. 2012), we find that the observed high polarization degree can only be achieved if the magnetic field direction is almost on the plane of the sky (Fig. 3). However, in this case, the model fails to reproduce both the low $\left(I / I_{\max }<0.4\right)$ and high $\left(I / I_{\max }>0.8\right)$ values of the normalized intensity. This suggests a higher/lower polarization efficiency at low/high normalized intensity values. To test this, we assumed a scenario with a constant but higher polarization efficiency, $\alpha=0.18$, up to a certain density $\left(n_{\text {depol }}\right)$ after which $\alpha$ becomes zero. The observational data can be well reproduced for a depolarization density of $n_{\text {depol }}=6 \times 10^{4} \mathrm{~cm}^{-3}$ (model 3 in Fig. 3 ), which corresponds to a visual extinction limit of $30 \mathrm{mag}$. In brief, the Pipe-109 submm polarization suggests that the core's center is unpolarized. These results supports the radiative torques theory of grain alignment, which requires a source of photons to help the alignment (Lazarian \& Hoang 2007).

\section{Conclusions}

Our multi-wavelength data represent the most complete polarimetric observations ever performed toward a starless core. This investigation shows that the magnetic field has a reasonable uniform morphology from physical scales of $0.4 \mathrm{pc}$ down to $0.08 \mathrm{pc}$. However, a discontinuity between the optical/near-IR and the submm polarization can be seen in the mean field direction.

The absorption and emission polarization regimes are well correlated to the cloud and core components, respectively. While the optical and near-IR polarization do not vary significantly with the diffuse/low extinction gas $\left(A_{V}<9 \mathrm{mag}\right)$, the submm polarization falls dramatically toward the peak of extinction. The near-IR polarization is produced by both the cloud and the core, resulting in two distinct regimes of polarization efficiencies of the dust grains. The near-IR polarization of the core overlaps in extinction with the submm polarization, but the cause for the distinct polarization efficiency regimes between them is still unclear. Further work should be done to understand this issue. The submm regime shows a steep decrease in polarization efficiency.
The lack of an internal source of radiation results in a loss of grain alignment with the magnetic field. This result is consistent with the theory of dust grain alignment via radiative torques.

The submm polarization is well reproduced by a magnetized, isolated core model, whose magnetic field is projected onto the plane of sky. The observed depolarization occurs for volume densities higher than $6 \times 10^{4} \mathrm{~cm}^{-3}$ (or $A_{V}>30 \mathrm{mag}$ ).

Acknowledgements. The authors would like to thank the APEX staff for the pool observations and the OPD staff for helping with the optical and nearIR observations. The authors would also like to thank Carlos Román-Zúñiga for providing the visual extinction map. F.O.A. acknowledges financial support from the BMBF Verbundforschung 05A11PD3 project. G.A.P.F. and F.P.S. acknowledge support from the Brazilian agencies CNPq, CAPES and FAPEMIG. F.P.S. is supported by the CAPES grant 2397/13-7. P.F. is supported by the Spanish CONSOLIDER project CSD2009-00038. P.F. and J.M.G. are supported by the Spanish MINECO AYA2011-30228-C03-02, and Catalan AGAUR 2009SGR1172 grants.

\section{References}

Allen, A., Li, Z.-Y., \& Shu, F. H. 2003a, ApJ, 599, 363

Allen, A., Shu, F. H., \& Li, Z.-Y. 2003b, ApJ, 599, 351

Alves, F. O., Franco, G. A. P., \& Girart, J. M. 2008, A\&A, 486, L13

Alves, F. O., Acosta-Pulido, J. A., Girart, J. M., Franco, G. A. P., \& López, R. 2011, AJ, 142, 33

Alves, F. O., Vlemmings, W. H. T., Girart, J. M., \& Torrelles, J. M. 2012, A\&A, 542, A14

Alves, J., Lombardi, M., \& Lada, C. J. 2007, A\&A, 462, L17

Andersson, B.-G., Pintado, O., Potter, S. B., Straižys, V., \& Charcos-Llorens, M. 2011, A\&A, 534, A19

Arce, H. G., Goodman, A. A., Bastien, P., Manset, N., \& Sumner, M. 1998, ApJ, 499, L93

Chapman, N. L., Goldsmith, P. F., Pineda, J. L., et al. 2011, ApJ, 741, 21

Crutcher, R. M., Nutter, D. J., Ward-Thompson, D., \& Kirk, J. M. 2004, ApJ, 600, 279

Dolginov, A. Z., \& Mytrophanov, I. G. 1976, Ap\&SS, 43, 257

Draine, B. T., \& Weingartner, J. C. 1996, ApJ, 470, 551

Draine, B. T., \& Weingartner, J. C. 1997, ApJ, 480, 633

Feitzinger, J. V., \& Stuewe, J. A. 1984, A\&AS, 58, 365

Fiege, J. D., \& Pudritz, R. E. 2000, ApJ, 544, 830

Forbrich, J., Lada, C. J., Muench, A. A., Alves, J., \& Lombardi, M. 2009, ApJ, 704, 292

Franco, G. A. P., Alves, F. O., \& Girart, J. M. 2010, ApJ, 723, 146

Frau, P., Girart, J. M., Beltrán, M. T., et al. 2010, ApJ, 723, 1665

Frau, P., Galli, D., \& Girart, J. M. 2011, A\&A, 535, A44

Frau, P., Girart, J. M., \& Beltrán, M. T. 2012a, A\&A, 537, L9

Frau, P., Girart, J. M., Beltrán, M. T., et al. 2012b, ApJ, 759, 3

Goodman, A. A., Jones, T. J., Lada, E. A., \& Myers, P. C. 1995, ApJ, 448, 748

Hall, J. S. 1949, Science, 109, 166

Hiltner, W. A. 1949, ApJ, 109, 471

Lazarian, A. 2007, J. Quant. Spectr. Rad. Transf., 106, 225

Lazarian, A., \& Hoang, T. 2007, MNRAS, 378, 910

Li, Z.-Y., \& Shu, F. H. 1996, ApJ, 472, 211

Magalhães, A. M., Rodrigues, C. V., Margoniner, V. E., Pereyra, A., \& Heathcote, S. 1996, in Polarimetry of the Interstellar Medium, eds. W. G. Roberge, \& D. C. B. Whittet, ASP Conf. Ser., 97, 118

Nutter, D. J., Ward-Thompson, D., Crutcher, R. M., \& Kirk, J. M. 2004, Ap\&SS, 292, 179

Padovani, M., Brinch, C., Girart, J. M., et al. 2012, A\&A, 543, A16

Rathborne, J. M., Lada, C. J., Muench, A. A., Alves, J. F., \& Lombardi, M. 2008, ApJS, 174, 396

Román-Zúñiga, C. G., Alves, J. F., Lada, C. J., \& Lombardi, M. 2010, ApJ, 725, 2232

Serkowski, K., Mathewson, D. S., \& Ford, V. L. 1975, ApJ, 196, 261

Siringo, G., Kreysa, E., Reichertz, L. A., \& Menten, K. M. 2004, A\&A, 422, 751

Siringo, G., Kovács, A., Kreysa, E., et al. 2012, in Proc. of the SPIE, Conf. Ser., 8452

Ward-Thompson, D., Kirk, J. M., Crutcher, R. M., et al. 2000, ApJ, 537, L135

Ward-Thompson, D., Sen, A. K., Kirk, J. M., \& Nutter, D. 2009, MNRAS, 398, 394

Whittet, D. C. B., Hough, J. H., Lazarian, A., \& Hoang, T. 2008, ApJ, 674, 304

Wiesemeyer, H., Hezareh, T., Kreysa, E., et al. 2014 [arXiv: 1408.5100] 